\title{
Correction to: Surface Stress Effects in Nanostructured Si Anode Particles of Lithium-ion Batteries
}

Amrita Sengupta, Sourav Das, and Jeevanjyoti Chakraborty

\section{Correction to:}

Chapter "Surface stress effects in nanostructured $\mathrm{Si}$ anode particles of Lithium-ion batteries" in: S. K. Saha and M. Mukherjee (eds.), Recent Advances in Computational Mechanics and Simulations, Lecture Notes in Mechanical Engineering, https://doi.org/10.1007/978-981-15-8315-5_4

In the original version of the book, the title of Chapter has been changed from "Surface Stress-induced Degradation of Electrochemical Performance of Cylindrical Silicon Anode Particles in Li-ion Batteries" to "Surface stress effects in nanostructured Si anode particles of Lithium-ion batteries". The erratum chapter and the book have been updated with the change. 